\title{
La alfabetización digital en España como medio de inclusión social: aplicación de un modelo de medi- ción a través de indicadores.
}

\author{
Mercedes CARIDAD-SEBASTIÁN \\ Universidad Carlos III de Madrid \\ mercedes@bib.uc3m.es \\ Ana María Morales-GarcíA. \\ Universidad Carlos III de Madrid \\ amorales@bib.uc3m.es \\ Fátima GARCÍA-LóPEZ. \\ Universidad Carlos III de Madrid \\ fatimag@bib.uc3m.es
}

\begin{abstract}
Resumen
A partir de la aplicación del modelo de medición desarrollado en el proyecto IMPOLIS, se presenta un análisis de los datos sobre el impacto real de las políticas TIC establecidas en relación a los aspectos de inclusión social.

El Proyecto IMPOLIS tiene como objeto de investigación elaborar un modelo de medición del impacto de las políticas TIC, que pueda ser empleado con el fin de orientar una toma de decisiones eficiente para el desarrollo armónico y sostenible de la Sociedad del Conocimiento. En esta investigación se seleccionaron las comunidades autónomas con menor PIB per cápita del territorio español, en relación a los aspectos especificados en el proyecto sobre la eficacia de las políticas TIC, y una muestra representativa de los grupos meta (mujeres, tercera edad, desempleados, discapacitados, población aquejada de analfabetismo, población inmigrante) por áreas geográficas.

El cuadro de mando de indicadores (scoreboard) correspondiente al modelo, se centra en tres categorías o dimensiones de estudio, Infraestructura Social TIC; Apropiación competencial y Nivel competencial, a su vez divididas en diversas subcategorías de análisis.

Los resultados obtenidos en este estudio permiten una primera contrastación de la validez del modelo de medición propuesto por el Proyecto IMPLOLIS, como instrumento de seguimiento de las políticas TIC. Se concluye que el método propuesto permite diseñar y redirigir medidas capaces de reducir la brecha digital, consolidando un modelo de inclusión social mediante la inclusión digital en consonancia con las actuales medidas de la Unión Europea.
\end{abstract}

Palabras Clave: Políticas TIC, Inclusión social, Indicadores, Scoreboard, España

\section{Digital literacy in Spain as a means of social inclusion: implementation of a measurement model using indicators.}

\begin{abstract}
:
Through the application of the measurement model developed in the project IMPOLIS, an analysis of data on the impact of ICT policies established in relation to issues of social inclusion is proposed.

The IMPOLIS research project aims to develop a model for measuring the impact of ICT policies, that can be employed to guide efficient decision making for harmonious and sustainable development of the Knowledge Society . In this research the regions with the lowest GDP per capita in the Spanish territory
\end{abstract}


were selected, in relation to the matters specified in the project on the effectiveness of ICT policies, and a representative sample of the target groups (women, elderly, unemployed, disabled, people suffering from illiteracy, immigrants) by geographical areas.

The scorecard indicators ( scoreboard ) for the model focuses on three categories or dimensions of study, Social Infrastructure ICT Competence and Level Appropriation Competence, in turn divided into several sub-categories of analysis.

The results obtained in this study allow a first checking of the validity of the proposed measurement proposed by the IMPLOLIS Project, as a monitoring tool for ICT policies.

The proposed method allows to design and redirect measures for reducing the digital divide, and to consolidate a model of social inclusion through digital inclusion in line with current EU measures .

Keywords: ICT Policies, Social Inclusion, Indicators, Scoreboard, Spain

\section{Referencia normalizada:}

Caridad Sebastián, M.; Morales García, A. M.; García López, F. (2013) La alfabetización digital en España como medio de inclusión social: aplicación de un modelo de medición a través de indicadores. Historia y Comunicación Social. Vol. 18 N Especial Octubre. Págs. 455-

\section{Sumario}

1. Introducción 2. Metodología 3. Resultados del estudio 3.1. Características sociodemográficas y socioeconómicas de los grupos meta 3.2. Infraestructura social tic: disponibilidad, intensidad, finalidad de usos y barreras al uso 3. 3. Apropiación COMPETENCial 3.4. Nivel competencial 4. Conclusiones 5.Bibliografia

\section{Introducción}

El proyecto "IMPOLIS: Indicadores para la medición del impacto en políticas TIC hacia la inclusión social”, financiado por el Ministerio de Ciencia e Innovación, ha permitido elaborar y testar un modelo de medición del impacto de las políticas TIC, que pueda ser empleado con el fin de orientar a una toma de decisiones eficiente para el desarrollo armónico, inclusivo y sostenible de la Sociedad del Conocimiento.

En consonancia con los objetivos propuestos en la Fase 5 del proyecto, "Análisis comparativo de resultados y propuestas de acción" en el presente estudio se ha realizado un análisis de los datos obtenidos que nos ha permitido testar la eficacia del modelo de evaluación y realizar propuestas de actuación en relación a las políticas públicas encaminadas a fomentar la inclusión social a través de las TIC.

\section{Metodología}

En la Fase 1 del proyecto IMPOLIS, se detectan y proponen los grupos meta en riesgo de exclusión (mujeres, tercera edad, desempleados, discapacitados, población aquejada de analfabetismo, población inmigrante), sobre los que descansará la propuesta de un Modelo Evaluativo para medir la efectividad de las políticas TIC para la inclusión digital y social. 
Para verificar la validez del modelo, en esta investigación se seleccionaron las dos comunidades autónomas (CCAA) con menor PIB per cápita del territorio español: Extremadura $(\mathrm{PIB}=15,3)$ y Andalucía $(\mathrm{PIB}=16,9)(\mathrm{INE}, 2012)$. Dado que los Telecentros, son espacios públicos que permiten el libre acceso de la ciudadanía a las TIC, se consideró que serían los lugares más idóneos para realizar la investigación en las CCAA seleccionadas. El número de telecentros por comunidad autónoma se calculó en función del porcentaje de población de cada una de ellas con respecto al total. Finalmente, participaron 19 telecentros, medida proporcionalmente ajustada al número de habitantes de las dos autonomías: 16 telecentros en Andalucía y 3 en Extremadura.

Las encuestas se realizaron a lo largo del año 2012. En cuanto al tamaño de la muestra, se tuvo en cuenta las recomendaciones de la bibliografía sobre metodología de la investigación social con respecto a una encuesta piloto. Finalmente, cumplimentaron la encuesta un $85,5 \%$ de la población prevista en la muestra, usuarios de un total de 19 telecentros, situados en poblaciones menores de 5.000 habitantes dentro de las dos comunidades autónomas.

Asimismo, en estas fases, se diseñaron dos instrumentos principales para el procesamiento estadístico de los datos obtenidos. Por un lado, el programa Microsoft Excel, y por otro la herramienta online de gestión de encuestas, Encuestafacil. com, que permitió el análisis de los resultados y la obtención de informes con barras de frecuencia y porcentajes, aplicar filtros a los informes, consultas cuestionario a cuestionario y otras funcionalidades para el procesamiento estadístico de los datos obtenidos.

\section{Resultados del estudio}

Mostramos a continuación los principales resultados del estudio agrupados en torno a los siguientes ámbitos de evaluación: Características sociodemográficas y socioeconómicas de los grupos meta; Infraestructura social TIC: disponibilidad, intensidad, finalidad de usos y barreras al uso; Apropiación competencial y Nivel competencial.

3.1. Características sociodemográficas y socioeconómicas de los grupos meta

Se ofrecen datos relativos a las características sociodemográficas y socioeconómicas de los grupos meta, atendiendo a diferentes variables como sexo, nacionalidad, situación económica y nivel de instrucción.

\section{Características sociodemográficas y socioeconómicas}

\begin{tabular}{|l|l|}
\hline Sexo & $\begin{array}{l}\text { El sexo es una variable trasversal a cualquiera de los grupos meta objeto } \\
\text { del estudio. En el estudio realizado, de un total de personas encuestadas, } \\
\text { el } 48,19 \% \text { corresponde al sexo femenino y el } 51,81 \% \text { al masculino, lo que } \\
\text { permite obtener una muestra similar de ambos sexos. }\end{array}$ \\
\hline
\end{tabular}




\begin{tabular}{|c|c|}
\hline \multicolumn{2}{|c|}{ Características sociodemográficas y socioeconómicas } \\
\hline Edad & $\begin{array}{l}\text { La muestra está compuesta por } 5 \text { rangos de edades. El } 45,7 \% \text { de las personas } \\
\text { que han cumplimentado la encuesta corresponde al grupo de edades } \\
\text { comprendidas entre } 31 \text { y } 50 \text { años, seguido del grupo de entre } 16 \text { y } 30 \text { años } \\
(31,33 \%) \text {, a continuación los de } 51 \text { a } 65 \text { años }(16,27 \%) \text {, de } 66 \text { a } 75(6,02 \%) \text { y } \\
\text { finalmente los mayores de } 76 \text { años }(0,6 \%) \text {. } \\
\text { Es importante destacar que la tipología de usuarios que más presencia tiene } \\
\text { en esta encuesta es el grupo de edades comprendidas entre } 31 \text { y } 50 \text { años. } \\
\text { La razón puede estar vinculada a que son los ciudadanos que más utilizan } \\
\text { el telecentro, como centro de acceso gratuito a Internet y como una posible } \\
\text { fuente de empleo. }\end{array}$ \\
\hline $\begin{array}{l}\text { Tipo de } \\
\text { discapacidad }\end{array}$ & $\begin{array}{l}\text { Por su repercusión en cuanto a la accesibilidad y demostración de la } \\
\text { capacidad solidaria de una comunidad política se decidió introducir, dentro } \\
\text { del grupo meta a los discapacitados. Del total de encuestados, el } 94 \% \text { no } \\
\text { tenía ningún tipo de discapacidad, el } 5 \% \text { señaló una discapacidad física y un } \\
1 \% \text { problemas de aprendizaje y discapacidad sensorial, respectivamente. }\end{array}$ \\
\hline Nacionalidad & $\begin{array}{l}\text { Pese a la importancia que reviste el colectivo de inmigrantes, sólo el } 8,43 \% \\
\text { de los encuestados son de origen extranjero. Este dato puede indicar el bajo } \\
\text { nivel de participación de los inmigrantes en las actividades que desarrollan } \\
\text { los telecentros. }\end{array}$ \\
\hline $\begin{array}{l}\text { Continente } \\
\text { y país de } \\
\text { nacimiento }\end{array}$ & $\begin{array}{l}\text { Del } 8,43 \% \text { de los encuestados de origen extranjero, los dos primeros lugares } \\
\text { corresponden a las personas de origen africano (Marruecos y Chad, con } \\
\text { un } 6,02 \% \text { y de países de América (Colombia y Ecuador, con un } 1,81 \% \text { ), } \\
\text { seguido de Oceanía (Australia, con un } 0,6 \% \text { ). }\end{array}$ \\
\hline $\begin{array}{l}\text { Estudios } \\
\text { terminados }\end{array}$ & $\begin{array}{l}\text { Se constata el bajo nivel de formación de las personas encuestadas. Un } \\
45,58 \% \text { corresponde a los niveles de estudios secundarios }(22,29 \%) \text { y de } \\
\text { Formación Profesional }(22,29 \%) \text {. } \\
\text { Es significativo el elevado porcentaje }(37,95 \%) \text { de personas con estudios } \\
\text { primarios }(30,72 \% \text { y sin estudios }(7,23 \%) \text {. Sólo un } 16,72 \% \text { corresponde a } \\
\text { ciudadanos con estudios superiores. }\end{array}$ \\
\hline $\begin{array}{l}\text { Situación } \\
\text { laboral }\end{array}$ & $\begin{array}{l}\text { En las comunidades autónomas españolas, la tasa de paro oscila entre el } \\
35,79 \% \text { de Andalucía y } 15,46 \% \text { en el País Vasco. El mayor porcentaje, } \\
\text { después de Andalucía, se registra en las Comunidades de Extremadura y } \\
\text { Canarias, con un } 33,69 \% \text { (EPA, 2013). } \\
\\
\text { Estos datos se reflejan también en nuestro estudio. Las dos Comunidades } \\
\text { Autónomas (Extremadura y Andalucía) muestran un alto porcentaje de } \\
\text { personas desempleadas ( } 21,08 \%) \text {, que unido con el grupo de estudiantes } \\
(17,47 \%) \text {, personas que realizan labores del hogar }(9,64 \%) \text { y otras } \\
\text { actividades }(3,61 \%) \text {, suman un total de } 51,80 \% \text { de personal sin actividad } \\
\text { laboral remunerada, frente al } 37,95 \% \text { de personal con vinculación laboral } \\
\text { (trabajadores por cuenta ajena, con un } 33,13 \% \text {, y trabajadores por cuenta } \\
\text { propia, con un } 4,82 \%) \text {. }\end{array}$ \\
\hline $\begin{array}{l}\text { Ingresos } \\
\text { mensuales }\end{array}$ & $\begin{array}{l}\text { Se constata el bajo nivel de ingreso de la población encuestada, el } 41,57 \% \\
\text { tienen ingresos de menos de } 1.100 \text { euros y un } 25,9 \% \text { no especifica sus } \\
\text { ingresos. Posiblemente esta última cifra pueda incluir a personas con bajo } \\
\text { o ningún tipo de ingreso, aspecto que elevaría la cifra a un } 67,47 \% \text {. Solo } \\
\text { un } 32,54 \% \text { ingresa un salario por encima de los } 1.100 \text { euros (un } 24,10 \% \text { de } \\
1.100-1.800 \text { euros; un } 6,63 \% \text { de } 1.800-2.700 \text { euros y únicamente } 1,81 \% \text {, más } \\
\text { de } 2.700 \text { euros). }\end{array}$ \\
\hline
\end{tabular}


3.2. Infraestructura social tic: disponibilidad, intensidad, finalidad de usos y barreras al uso

Se muestran en este apartado resultados relativos a la disponibilidad, fines y usos de las TIC por parte de los grupos meta.

3.2.1. Disponibilidad e intensidad: porcentaje de individuos que utilizan las TIC, contenidos y servicios

Se constató que los usuarios hacían un uso elevado de los ordenadores, con un $97,66 \%$. En cuanto a la frecuencia, pudimos detectar que un $65,87 \%$, lo usaba diariamente, mientras que un $25,15 \%$, sólo, una vez por semana.

Si comparamos el uso de los ordenadores con el acceso a Internet, observamos datos similares. El porcentaje de acceso a Internet es del 96,49\%, con una frecuencia de conexión diaria del $64,07 \%$, seguido de un $25,15 \%$ de usuarios que acceden, al menos, una vez por semana. Por debajo del $10 \%(9,78 \%)$, se agrupan las respuestas que afirman que esporádicamente acceden a Internet $(9,58 \%)$ o que no acceden nunca $(1,2 \%)$.

3.2.2. Barreras de uso: porcentaje de individuos que no utilizan las TIC, sus contenidos y servicios (tanto físico, como virtuales)

De los resultados obtenidos, se deduce que la razón principal por la que no usaban los ordenadores e Internet está vinculada fundamentalmente a que un $57,14 \%$ de los que aún no lo utilizan, no saben cómo acceder a ellos y un 28,57\% manifiesta que se encuentra en proceso de aprendizaje, o no se lo propone por falta de tiempo. Es importante señalar que un $14,29 \%$ de los encuestados afirma que el uso de las TIC no representa una herramienta útil para su vida cotidiana.

3.2.3. Finalidad de uso: porcentaje de individuos que utilizan las TIC, contenidos y servicios para diferentes fines

Queda patente la importancia que ejerce el acto de comunicarse para los ciudadanos encuestados (80\%), siendo ésta la finalidad primordial en el uso de las TIC (tabla 1). Independientemente de la región de origen, los usuarios, no dudan en aprender y utilizar las TIC para relacionarse y contactar con familiares, amigos, compañeros de trabajo y otros internautas.

\begin{tabular}{|l|c|c|c|}
\hline Actividades (Finalidad de uso) & $\begin{array}{c}\text { No lo } \\
\text { uso }\end{array}$ & $\begin{array}{c}\text { Necesito } \\
\text { ayuda }\end{array}$ & $\begin{array}{c}\text { Me manejo } \\
\text { con soltura }\end{array}$ \\
\hline Me comunico con mi familia, amistades o trabajo & $7 \%$ & $13 \%$ & $80 \%$ \\
\hline Me informo de la actualidad & $14 \%$ & $13 \%$ & $72 \%$ \\
\hline Redes sociales & $13 \%$ & $14 \%$ & $72 \%$ \\
\hline $\begin{array}{l}\text { Escribo cartas, informes o trabajos de clase, con Word } \\
\text { o similar }\end{array}$ & $20 \%$ & $18 \%$ & $62 \%$ \\
\hline
\end{tabular}




\begin{tabular}{|l|c|c|c|}
\hline Actividades (Finalidad de uso) & $\begin{array}{c}\text { No lo } \\
\text { uso }\end{array}$ & $\begin{array}{c}\text { Necesito } \\
\text { ayuda }\end{array}$ & $\begin{array}{c}\text { Me manejo } \\
\text { con soltura }\end{array}$ \\
\hline $\begin{array}{l}\text { Realizo actividades de ocio como juegos, sacar entradas, } \\
\text { viajes, música... }\end{array}$ & $25 \%$ & $20 \%$ & $56 \%$ \\
\hline Me informo sobre temas relacionados con la salud & $43 \%$ & $13 \%$ & $44 \%$ \\
\hline Hago cursos de formación & $38 \%$ & $20 \%$ & $43 \%$ \\
\hline Realizo gestiones con la administración, bancos, etc. & $47 \%$ & $15 \%$ & $38 \%$ \\
\hline Busco trabajo & $49 \%$ & $14 \%$ & $37 \%$ \\
\hline Hago compras por Internet & $50 \%$ & $14 \%$ & $36 \%$ \\
\hline $\begin{array}{l}\text { Edito imágenes, video o audio con programas como } \\
\text { Photoshop }\end{array}$ & $45 \%$ & $22 \%$ & $34 \%$ \\
\hline $\begin{array}{l}\text { Utilizo programas de presentaciones para mi trabajo } \\
\text { como Power Point }\end{array}$ & $46 \%$ & $22 \%$ & $32 \%$ \\
\hline $\begin{array}{l}\text { Uso hojas de cálculo para la contabilidad del hogar o en } \\
\text { mi trabajo, como Excel }\end{array}$ & $51 \%$ & $20 \%$ & $28 \%$ \\
\hline Actualizo y mantengo bases de datos como Access & $59 \%$ & $19 \%$ & $22 \%$ \\
\hline
\end{tabular}

Tabla 1: Finalidad de uso Fuente: Elaboración propia

El segundo ítem, en cuanto a finalidad de uso, está relacionado con la lectura de noticas de actualidad. Llama la atención que un 72\% de los encuestados utilizara Internet para estar informado (tabla 1).

Con el mismo porcentaje (72\%), los internautas encuestados manifiestan hacer uso de redes sociales. Este medio de comunicación está demostrando, cada vez más, su popularidad entre la población, independientemente de su estatus socioeconómico (tabla 1).

El uso de Internet está también muy asociado a las actividades de ocio. Un $\mathbf{5 6 \%}$ de encuestados realizó diferentes actividades relacionadas con juegos, sacar entradas, viajes, música, entre otras. Llama la atención el elevado porcentaje que manifiesta hacer uso de esta actividad por encima de otras de carácter profesional o informacional (formación, administración pública, salud y trabajo) (tabla 1), las cuales oscilan entre un 44\% y $37 \%$. Este es el caso de búsqueda de información sobre salud (44\%), cursos de formación (43\%), gestiones de la administración (38\%) y de trabajo (37\%). Sin embargo, el acto de realizar compras, en general, a través de Internet se sitúa en un 36\%. Esta actividad entendida como comercio electrónico en su sentido más amplio, y que abarca no solo el pago de entradas o viajes, aún crea recelo entre los ciudadanos españoles (tabla 1).

Finalmente, mientras que actividades relacionadas con la redacción de cartas, informes o trabajos de clase, obtuvieron un $62 \%$, el uso de herramientas ofimáticas (para la edición de imágenes, video o audio, el uso programas de presentaciones para el trabajo, hojas de cálculo para la contabilidad del hogar o en el trabajo y el acceso a bases de datos), se sitúa entre un 34\% y un 22\% del total (tabla 1). 
3.2.4. Disponibilidad: porcentaje de bibliotecas, telecentros, puntos de información, centros cívicos y sociales financiados con fondos públicos que permiten acceder a las TIC

Se pudo detectar que sólo el $28,14 \%$ de los usuarios que asistían a los telecentros, tenían noción de otros puntos de acceso público a Internet gratuitos, como bibliotecas y centros culturales.

Por lo que respecta a las bibliotecas, la explicación de este porcentaje tan bajo podría estar relacionada con el hecho de que el estudio se ha realizado en municipios con menos de 5.000 habitantes, aspecto que no obliga legalmente a que el municipio cuente con una biblioteca pública.

\subsection{Apropiación competencial}

Este apartado pretende obtener datos para conocer el nivel de formación en TIC de las personas que participan en el estudio.

3.3.1. Medios utilizados: porcentaje de individuos que afirman conocer el uso de las TIC, sus contenidos y servicios a partir de la información recibida a través de diferentes medios

Los encuestados manifiestan haber tenido conocimiento del uso de los ordenadores e Internet a través de diferentes medios: familiares, amistades o trabajo (91\%), los Punto de Acceso Público a internet: bibliotecas, telecentros, centros culturales, etc. (84\%), seguido de los colegios, institutos u otros centros educativos (68\%), los cursos específicos (64\%) y por último los medios de comunicación (Televisión, con 53\%, y Radio, con un $35 \%$ ) (gráfico 1 ).

Gráfico 1: Internet y los ordenadores: medios e instituciones que intervienen para su conocimiento.

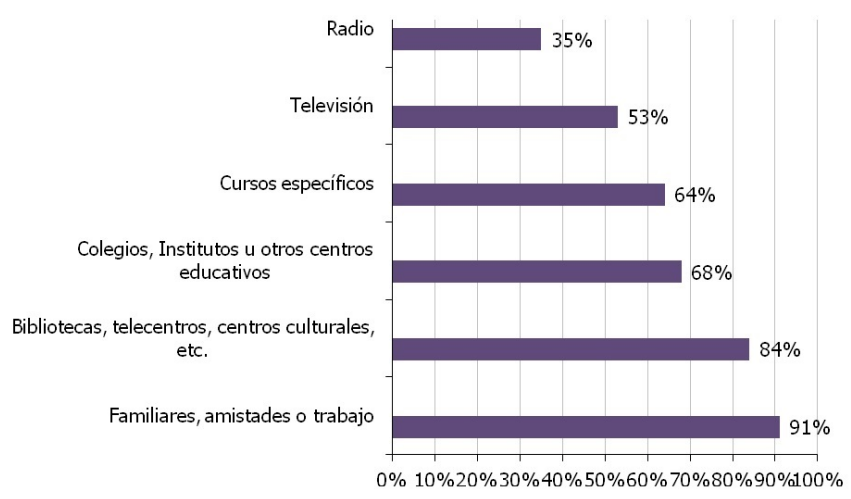

Fuente: Elaboración propia 
3.3.2. Finalidad de la participación en actividades formativas TIC: porcentaje de individuos que afirman formarse en TIC, sus contenidos y servicios

El $65 \%$ de los encuestados han manifestado que han participado en cursos relacionados con la alfabetización digital, lo que denota un uso avanzado de los ordenadores e Internet. De este porcentaje de internautas, un $81 \%$ señala que prioritariamente realizan estos cursos para incrementar sus posibilidades de ocio y cultura, un $77 \%$ para relacionarse con otras personas, un $70 \%$ para incrementar sus posibilidades de empleo, un $67 \%$ para hacer gestiones con la administración y un 55\% para usar los servicios de salud de atención primaria (gráfico 2).

Gráfico 2: Motivos para la participación en cursos de alfabetización digital.

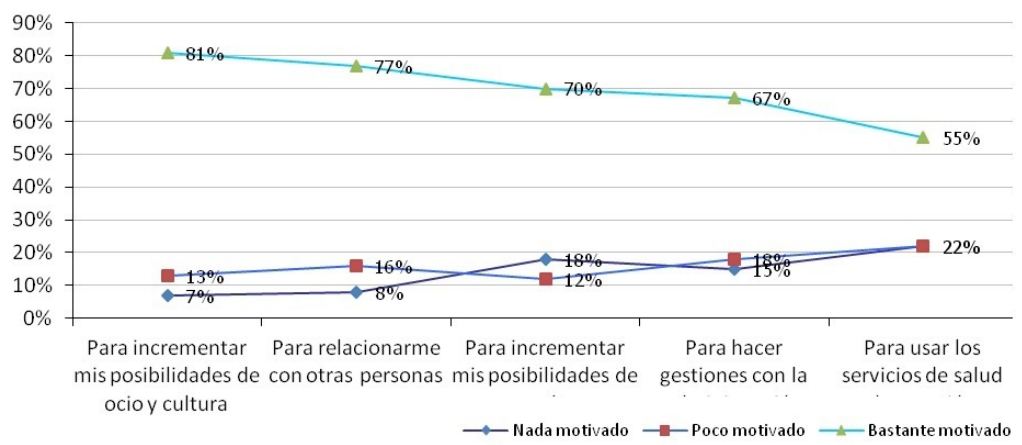

Fuente: Elaboración propia

3.3.3. Intensidad: porcentaje de individuos que afirman haber participado en actividades de formación encaminadas a saber utilizar tecnologías, contenidos o servicios digitales

El 65\% de los encuestados han manifestado que han participado en cursos relacionados con la alfabetización digital. De este total, un $62,14 \%$ suscribe haber realizado cursos varias veces al año, seguido de un $24,27 \%$ que exponen que solo los efectúan esporádicamente, quedando sólo un $13 \%$ para aquellos internautas que los solicitan 1 vez al año.

3.3.4. Satisfacción: grado de satisfacción expresado con la formación recibida en los cursos TIC en que se ha participado

Del $65 \%$ de internautas que han participado en cursos relacionados con la alfabetización digital, un 56,31\% manifiesta estar muy satisfecho y un $41,75 \%$ bastante satisfecho con la realización de estos cursos. Solo un 1,94\% muestra su insatisfacción. 


\subsection{Nivel competencial}

Pretende obtener datos para conocer el nivel de manejo y uso de la información de las personas encuestadas.

3.4.1. Percepción: porcentaje de individuos que afirman haber resuelto cualquier tipo de problemas prácticos gracias a la información que han encontrado en Internet

La percepción de los encuestados sobre la capacidad para resolver problemas prácticos de la vida cotidiana a través de Internet es muy baja. El 56,26\% responde que sólo, en ocasiones, obtienen la información necesaria, junto al 7,41\% que manifiesta que nunca lo solucionan a través de este medio, lo que denota que, o bien, no se sienten muy seguros (por falta de formación), o bien, no lo valoran como fuente de información fiable para su vida cotidiana. Sólo el 33,33\% afirma que siempre resuelve problemas prácticos.

3.4.2. Nivel competencial: porcentaje de individuos que son capaces de trasladar su necesidad de información a los distintos servicios de información que utilizan, teniendo en cuenta las características que estos presentan (capacidad para encontrar información)

Es importante destacar el alto porcentaje de encuestados que, ante una necesidad de información, utilizan un buscador como Google $(61,73 \%)$ y el bajo acceso a fuentes más específicas o bases de datos especializadas. Únicamente un tercio de la muestra (35,8\%) manifiesta que dependiendo de lo que busca, utiliza los dos métodos (accede a un buscador como Google o teclea la dirección de la página que le interesa) y sólo el 2,47\% de los usuarios accede directamente al sitio Web.

3.4.3. Nivel competencial: porcentaje de individuos que son capaces de seleccionar, en las búsquedas que realizan, posibles fuentes de información a utilizar para solucionar su necesidad de información

Google, hoy por hoy, es uno de los buscadores más utilizados. Permite obtener resultados web y realizar búsquedas de imágenes, vídeos, blogs, noticias, artículos, libros y mapas.

En nuestra investigación se constata que un alto porcentaje de la muestra (un 90\%) de los usuarios que acceden a Google, plantean sus estrategias de búsqueda mediante el uso de palabras clave, sin utilizar otra sintaxis de búsqueda. De los cuales, el 31\% replantea los términos de búsqueda si no encuentran los resultados que le interesa y un $28 \%$ lee los primeros resultados y normalmente soluciona los problemas. Solo el $12 \%$ pide ayuda a un tercero. 
Gráfico 3: Búsqueda de información en Google.

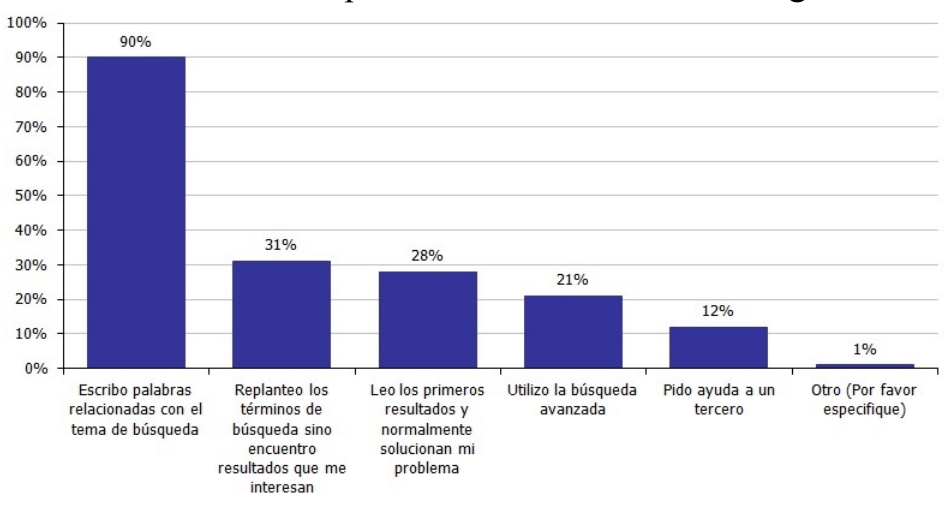

Fuente: Elaboración propia

El porcentaje de encuestados que recurre a otras opciones más avanzadas es de un $21 \%$, aspecto que denota su capacidad para trasladar sus necesidades informativas a estrategias de búsquedas que le permitan obtener respuestas más precisas y pertinentes. Estas estrategias también pueden incluirse, directamente, desde el cuadro de búsqueda de Google, si los usuarios conocen el sistema de búsqueda y todas sus posibilidades (gráfico 3).

Gráfico 4: Búsqueda de páginas conocidas.

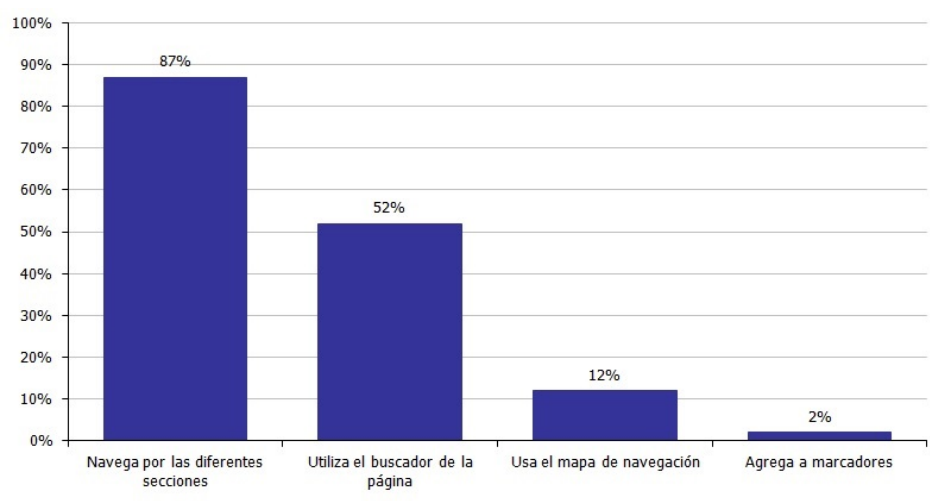

Fuente: Elaboración propia

Por lo que respecta a cómo los usuarios encuestados navegan por un sitio Web, un $87 \%$ afirma que se limita a navegar por las diferentes secciones, el 52\% utiliza el buscador de la página, un $12 \%$ usa el mapa de navegación y, únicamente, el $2 \%$ lo añade a marcadores (gráfico 4). 
3.4.4. Nivel competencial: porcentaje de individuos que utilizan distintas fuentes de información en función de su necesidad de información

Los usuarios encuestados manifiestan, mayoritariamente, que, no utilizan un sistema de alerta para mantenerse informado, mientras que un porcentaje elevado (69\%), afirma visitar directamente las páginas que le interesa de manera periódica (gráfico 5).

El segundo dato más significativo, corresponde al uso de las redes sociales. Un $53 \%$ utiliza este medio social para estar informado (gráfico 5). No es de extrañar este dato, teniendo en cuenta que uno de los cambios más relevantes de los últimos tiempos tiene que ver con la aparición de los medios sociales, como fuente de comunicación y de acceso a contenidos.

En cuanto a los sistemas de alertas, detectamos, nuevamente, la repercusión de las herramientas desarrolladas por Google. Un $41 \%$ de los encuestados afirma mantenerse informado a través de las alertas de Google, frente a un $10 \%$ que manifiesta recibir las últimas novedades por medio de RSS y listas de distribución, respectivamente (gráfico 5).

Gráfico 5: Sistemas de alertas y otros sistemas para mantenerse actualizados.

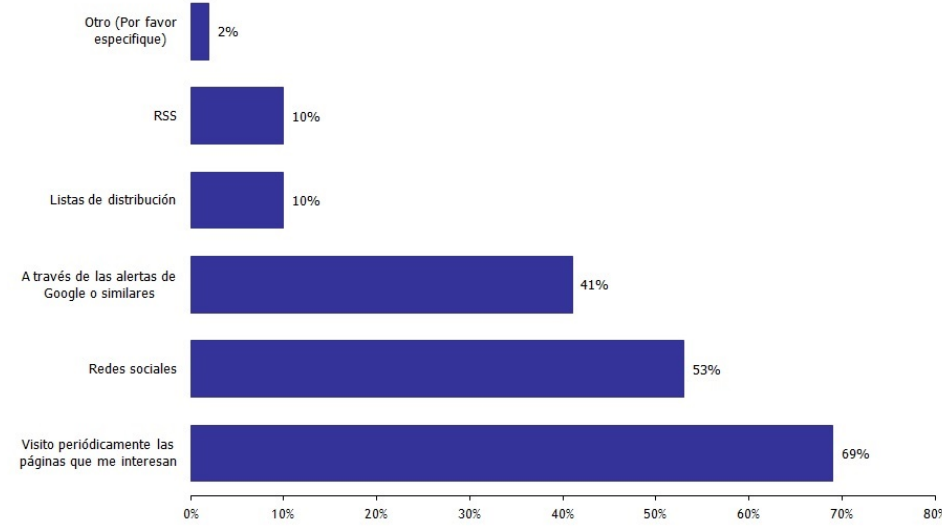

Fuente: Elaboración propia

3.4.5. Nivel competencial: porcentaje de individuos que son capaces de valorar la fiabilidad de la información que encuentran en los servicios de información digitales (capacidad para valorar la información)

Al indagar sobre la fiabilidad de la información que se encuentra en Internet, se constata que, únicamente, el 16\% confía en todo lo que encuentra en la Red y un 17\% reconoce el contenido sectario o engañoso (gráfico 6). 
Entre el $21 \%$ y el $42 \%$ del colectivo encuestado manifiesta que para valorar la información y cerciorarse de su validez:

- Examina y compara la información de varias fuentes (42\%).

- Intenta conocer quién ha escrito el contenido que ha consultando (39\%).

- Es muy selectivo en la información que utiliza (31\%).

- Le resulta más fiable la información si se citan fuentes utilizadas (23\%).

- Tiene en cuenta la fecha de actualización del contenido (21\%).

Gráfico 6: Capacidad para valorar la información.

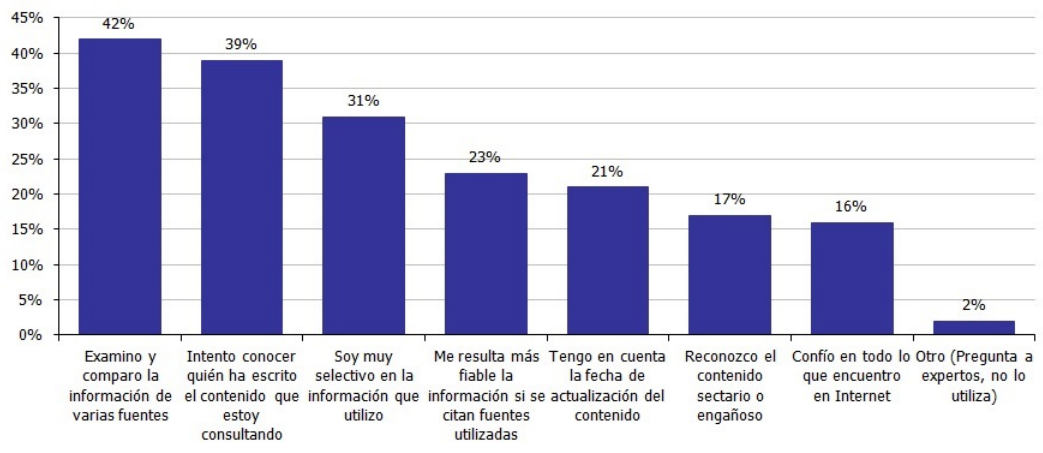

Fuente: Elaboración propia

3.4.6. Impacto: porcentaje de individuos que utilizando la información y los servicios disponibles en Internet afirman encontrar empleo, resolver problemas legales, de salud y administrativos

El grado de éxito obtenido en la búsqueda y resolución de problemas en áreas consideradas suficientemente representativas de la Sociedad de la Información muestra los siguientes datos: el $31 \%$ manifiesta haber resuelto problemas administrativos, el $21 \%$ problemas de salud, el $9 \%$ ha encontrado empleo a través de Internet y, finalmente, un $8 \%$ ha resuelto problemas legales (gráfico 7).

Es importante destacar que los beneficios que nos ofrece Internet en la búsqueda y resolución de problemas, no es lo suficientemente significativo para los grupos meta, objeto de estudio. Los datos obtenidos oscilan entre el 31\% y el $8 \%$ (gráfico 7). 
Gráfico 7: Grado de éxito obtenido en la búsqueda y resolución de problemas.

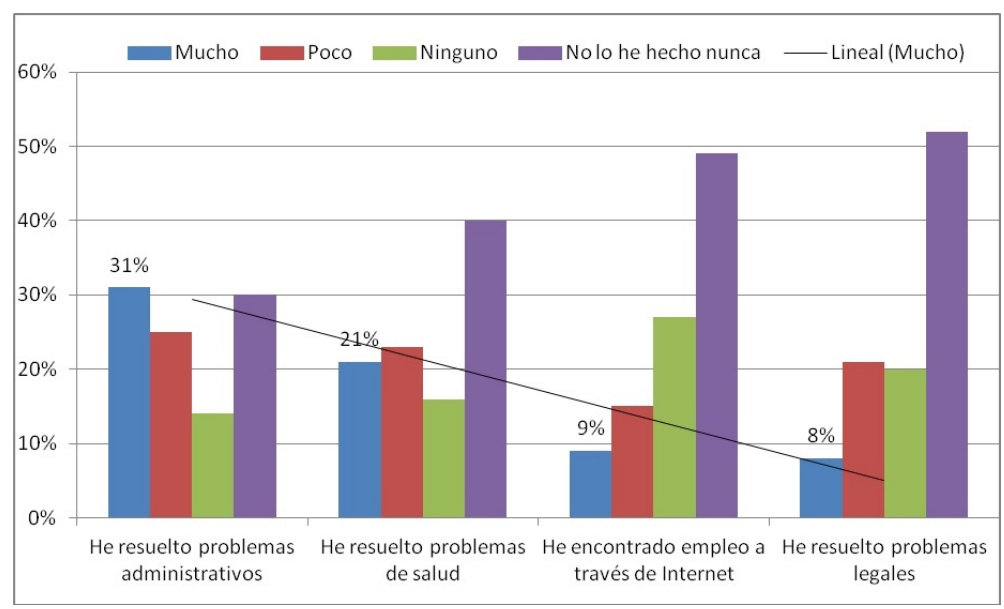

Fuente: Elaboración propia

3.4.7. Impacto: porcentaje de individuo que perciben como beneficioso el uso de las TIC, contenidos y servicios digitales

La percepción general en relación a las oportunidades que abre el uso de las TIC, sus contenidos y servicios para mejorar su calidad de vida, con independencia del nivel competencial que se posea, es bastante elevada. El 76,07\% afirma que el uso de las TIC ha influido significativamente en la mejora de la calidad de vida. Solo un $23,92 \%$ manifiestan que este uso ha influido poco o nada en la mejora de su calidad de vida.

\section{Conclusiones}

1. Se confirma la validez aplicativa del modelo propuesto, señalando algunas recomendaciones que ayudarían a completar la investigación:

Teniendo en cuenta las características tan dispares que pueden tener los diferentes grupos meta en cuanto a formación, conocimiento de las TICs y nivel económico sería conveniente crear una pregunta filtro que discrimine las respuestas por colectivos encuestados, lo que permitiría obtener datos independientes o discriminados de cada uno de los siete grupos metas: mujeres, tercera edad, desempleados, discapacitados, población inmigrante, personas que habitan en regiones desfavorecías y población aquejada de analfabetismo funcional y digital.

Puesto que el sexo, la edad, la condición social, el país de nacimiento, el nivel educativo o el estatus de empleo, pueden ser variables transversales a cualquiera de 
los grupos metas, objeto de estudio, se recomienda un segundo análisis independiente combinando características sociodemográficas, por ejemplo, mujeres desempleadas; desempleados discapacitados, inmigrantes en zonas desfavorecidas, etc.

Así mismo, sería conveniente en futuros estudios la colaboración con asociaciones y centros que atiendan a estos colectivos específicos: inmigrantes, discapacitados, mujeres, etc.

Por otra parte, sería relevante conocer el tiempo de residencia del inmigrante. Esto aportaría datos sobre su nivel de integración en la comunidad.

Para completar el estudio sería necesario identificar si el municipio cuenta con otros puntos de acceso a Internet gratuitos y los servicios desarrollados por estas entidades.

Finalmente, para poder realizar una comparativa por regiones o CCAA, sería recomendable una pregunta filtro que discrimine las respuestas de cada una de las áreas geográficas analizadas.

2. Este modelo de medición está orientado a la toma de decisión sobre futuras propuestas de acción destinadas a potenciar la inclusión social en España. El proyecto desarrollado está en consonancia con los retos de futuro y acciones estratégicas que prevé el Plan Avanza para el período 2011-2015: potenciar la aplicación de las TIC al sistema educativo y formativo e incrementar el uso avanzado de servicios digitales por la ciudadanía. La investigación subraya la relevancia que adquieren las competencias relativas al acceso, manejo y uso de la información, como medio para evitar que la diferencia existente en el grado de apropiación de las TIC, se convierta en un nuevo y poderoso "marcador de clase" en sectores de población ya de por sí vulnerables (grupos en riesgo de exclusión).

\section{Bibliografia}

CARIDAD SEBASTIÁN, Mercedes; MORALES GARCÍA, Ana María. "El papel de la sociedad de la información en el desarrollo científico". Crítica. 2007, año 57, no. 948, pp. 49-52.

CARIDAD SEBASTIÁN, Mercedes; MORALES GARCÍA, Ana María. "Las Políticas de Información europeas como acción clave para el desarrollo de la Ciencia de la Información". Ciência da Informação. v. 40, n. 3, 2011.

IMPOLIS: Indicadores para la medición del impacto en políticas TIC para la inclusión social. [PROYECTO DE INVESTIGACIÓN]. Investigador responsable: Mercedes Caridad; Entidad financiadora: MINISTERIO de Ciencia e Innovación. Duración: 2009-2012. Referencia: CSO2009-12291 (SUBPROGRAMA SOCI).

INE. Encuesta de Población Activa (EPA) (25 de julio de 2013). [7-10-2013]. Disponible en Internet: http://www.ine.es/daco/daco42/daco4211/epa0213.pdf 
INE. Producto Interior Bruto regional. Año 2012. [7-10-2013]. Disponible en Internet: http://www.ine.es/prensa/np774.pdf

MORALES GARCÍA, Ana María. La Sociedad de la Información: políticas y acciones en Europa. Bogotá : Rojas Eberhard, 2008.

MORALES GARCÍA, Ana María; CARIDAD SEBASTIÁN, Mercedes; GARCÍA LÓPEZ, Fátima. "The social impact and the competence of the spanish telecenters in the Information Society: evaluation methodology from indicators and multivariable methods of analysis". v.37, no .2, Brasília Mar./Aug. 2008. [7-102013]. Disponible en Internet: http://www.scielo.br/scielo.php?pid=S0100$19652008000200006 \&$ script $=$ sci_arttext

MORALES GARCÍA, Ana María; CARIDAD SEBASTIÁN, Mercedes; GARCÍA LÓPEZ, Fátima. "Telecentros en España: redes y contenidos". El profesional de la información, v. 18, $\mathrm{n}^{\mathrm{o}}$. 2, marzo-abril 2009. [7-10-2013]. Disponible en Internet: http://www.elprofesionaldelainformacion.com/Contenidos/2009/marzo/06.pdf

\section{Las autoras}

Mercedes Caridad-Sebastián es Doctora el Ciencias de la Información. Catedrática del Dpto. de Biblioteconomía y Documentación. Directora del Instituto Universitario "Agustín Millares" de Documentación y Gestión de la Información de la Universidad Carlos III de Madrid

Ana María Morales-García.es Doctora en Documentación. Profesora del Dpto. de Biblioteconomía y Documentación. Vice-decana del Campus de Colmenarejo. Miembro del Instituto Universitario “Agustín Millares" de Documentación y Gestión de la Información de la Universidad Carlos III de Madrid

Fátima García-López es Doctora en Documentación. Profesora del Dpto. de Biblioteconomía y Documentación. Secretaria Académica del Directora del Instituto Universitario "Agustín Millares" de Documentación y Gestión de la Información de la Universidad Carlos III de Madrid 